\title{
Dusty Structure Around Type 1 AGNs
}

\author{
Rivay Mor ${ }^{1}$, Hagai Netzer ${ }^{1}$, and Moshe Elitzur ${ }^{2}$ \\ ${ }^{1}$ Tel-Aviv University, Tel-Aviv 69978, Israel \\ Email: rivay@wise.tau.ac.il \\ ${ }^{2}$ University of Kentucky, Lexington, KY 40506-0055, USA
}

\begin{abstract}
We present a detailed investigation of the MIR continuum emission of type 1 QSOs and define the main emission components. Our model requires three components: a clumpy torus, dusty narrow-line region (NLR) clouds and a blackbody-like hot dust.
\end{abstract}

Keywords. galaxies: active, quasars: general

We fitted the $\sim 2-35 \mu \mathrm{m}$ spectra of 26 luminous ( $\left.\log L_{5100}=44.5-46\right)$ QSOs using Spitzer/IRS and ground-based NIR data. The models utilize the clumpy torus of Nenkova et al. (2008) and are the first to allow its consistent check in type 1 AGNs. Single torus models and combined torus-NLR models fail to fit the spectra of most sources but the addition of a third, hot BB component enables an adequate fit to all spectra. We present torus inclination, cloud distribution, covering factor and torus mass for all sources and compare them with $L_{\mathrm{bol}}, M_{\mathrm{BH}}$ and $L / L_{\mathrm{Edd}}$. The torus mass ranges from $4.5 \times 10^{-4}$ to $0.08 M_{\mathrm{BH}}$ and is correlated with $L_{\mathrm{bol}}$. The mean torus covering factor is 0.27 and it may also be anticorrelated with $L_{\mathrm{bol}}$, if some possibly anomalous points are omitted. We quantify the emission by the NLR clouds and estimate their distance from the center. The distances are $\sim 700$ times larger than the dust sublimation radius and the mean NLR covering factor is about 0.07 . We find that a substantial amount of the $\sim 2-7 \mu \mathrm{m}$ radiation originates from a hot dust component, which is likely situated in the innermost part of the torus. The luminosity radiated by this component and its covering factor are comparable to those of the torus. The total covering factor by all components is in good agreement with the known AGN type-1:type-2 ratio. Details are given by Mor et al. (2009).

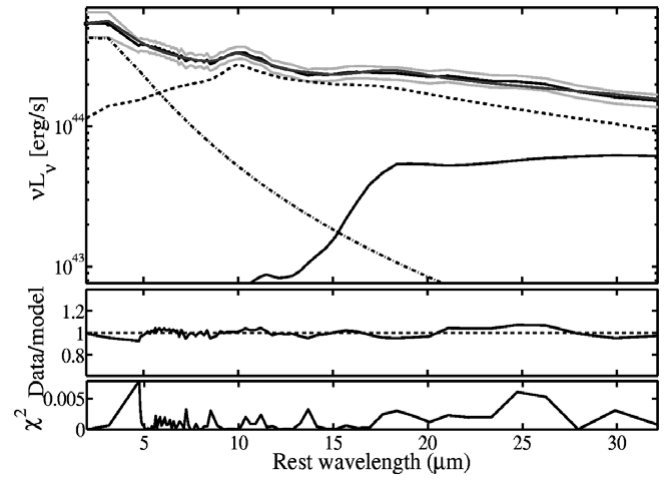

Figure 1. Three-component fit to the spectrum of PG 1617+175. Top: best fit model and the observed spectrum. We also show individual components: torus (dashed line), NLR (thick black line) and hot BB (dashdotted line). Bottom and middle panels show the quality of the fit. A color version of this figure is available online.

\section{References}

Mor, R., Netzer, H., \& Elitzur, M. 2009, ApJ, 705, 298

Nenkova, M., Sirocky, M. M., Ivezić, Ž., \& Elitzur, M. 2008, ApJ, 685, 147 